\title{
Periodic orbit bifurcations as an ionization mechanism: The bichromatically driven hydrogen atom
}

\author{
S. Huang ${ }^{1}$, C. Chandre ${ }^{2}$, T. Uzer ${ }^{1}$ \\ ${ }^{1}$ Center for Nonlinear Science, School of Physics, Georgia Institute of Technology, \\ Atlanta, Georgia 30332-0430, U.S.A. \\ ${ }^{2}$ Centre de Physique Théoriquet - CNRS, Luminy - Case 907, 13288 Marseille cedex \\ 09, France \\ E-mail: gtg098n@mail.gatech.edu
}

\begin{abstract}
We investigate the multiphoton ionization of hydrogen driven by a strong bichromatic microwave field. In a regime where classical and quantum simulations agree, periodic orbit analysis captures the mechanism : Through the linear stability of periodic orbits we match qualitatively the variation of experimental ionization rates with control parameters such as the amplitudes of the two modes of the field or their relative phases. Moreover, we discuss an empirical formula which reproduces quantum simulations to a high degree of accuracy. This quantitative agreement shows the mechanism by which short periodic orbits organize the dynamics in multiphoton ionization. We also analyze the effect of longer pulse durations. Finally we compare our results with those based on the peak amplitude rule. Both qualitative and quantitative analyses are implemented for different mode locked fields. In parameter space, the localization of the period doubling and halving allows one to predict the set of parameters (amplitudes and phase lag) where ionization occurs.
\end{abstract}

PACS numbers: $32.80 . \mathrm{Rm}, 05.45 .-\mathrm{a}$

Submitted to: J. Phys. B: At. Mol. Phys.

$\ddagger$ UMR 6207 of the CNRS, Aix-Marseille and Sud Toulon-Var Universities. Affiliated with the CNRS Research Federation FRUMAM (FR 2291). CEA registered research laboratory LRC DSM-06-35. 


\section{INTRODUCTION}

Among systems at the atomic level, one-electron systems are the most fundamental and have proven to be a source of numerous surprising discoveries [1] : The multiphoton ionization of hydrogen in a strong microwave field [2] is such a simple system with surprisingly complex dynamics. With the development of theory of chaos, its stochastic and diffusional nature was elucidated [3], and intense research activity in the last three decades has resulted in a rather complete understanding of this problem [4, 5, 6. In the recent years, the attention has shifted from understanding the physical process to manipulating or controlling it [7, 8]. In this context control refers to tailoring the physical behavior of dynamical systems (which generically show chaotic dynamics) using "knobs" (i.e., suitable external parameters). Identifying such knobs and understanding the mechanism by which they affect the dynamics is the ultimate goal of such research.

Bichromatic pulses [9, 10] are natural tools in atomic control research because they offer practical control parameters such as polarization, amplitudes and phases [11, 12, 15, 13, 14, 16, 17, 18, 19, 20, 21]. It has been shown that it is possible to use the direction of transport in a ratchet by varying the phase lag in the bichromatic pulse [22]. In this manuscript we consider the ionization behavior of a one-dimensional hydrogen atom driven by a strong bichromatic linearly polarized electric field which is modeled by the following one-dimensional Hamiltonian $(x>0)$ in atomic units

$$
H=\frac{p^{2}}{2}-\frac{1}{x}+F_{h} x \sin (h \omega t)+F_{l} x \sin (l \omega t+\phi),
$$

where the indices $l$ and $h$ refer to the low and high frequency modes with frequencies $l \omega$ and $h \omega$, respectively. These two modes are frequency locked (and denoted $h: l$ ), i.e. $l$ and $h$ are integers. The experimental results on ionization probability obtained in Refs. [17, 19] for two cases, mode lockings 3:1 and 3:2, show two very distinct regimes as the phase lag $\phi$ is varied : For 3:1, the ionization probability shows a plateau in phase located at a rather high value of ionization probability, and for 3:2, the ionization probability shows no such plateau but rather a small value of ionization probability. These results were confirmed by quantum calculations on the one-dimensional model (1).

The purpose of this manuscript is to show that these experimental observations are qualitatively and quantitatively captured using a periodic orbit analysis, which reveals the classical bifurcations responsible for ionization. Our analysis also allows the prediction of ionization at other values of parameters without resorting to large numerical simulations. A part of the results presented in this manuscript were announced in a Fast Track Communication [23].

This paper is organized as follows: First, in Sec. 2, we summarize the residue analysis of periodic orbits [24]. In Sec. 3] and Sec. 4, we consider two cases, the 3:1 mode locking and 3:2 one, respectively. These two cases show drastically different ionization behavior which we explain through bifurcations of selected periodic orbits. In these two cases, we described the bifurcations (if any) in the system. We also compute the bifurcation surface which is defined by the set of parameters where a change of linear 
stability (associated with a period doubling or halving) has occurred in the chaotic sea. In Sec. [5 we discuss the generalities of residue curve behavior for other mode lockings.

\section{Residue analysis of periodic orbits}

First, we map Hamiltonian (11) into action-angle variables (of the unperturbed system $\left.F_{h}=F_{l}=0\right)$ such that the principal quantum number $n$ is reflected by action $J$. The relationship between $J$ and $n$ reads

$$
J=\left(t_{0} \omega\right)^{1 / 3} n,
$$

where $t_{0}=2.4188843243 \times 10^{-17} \mathrm{~s}$ (atomic time unit) and $\omega=2 \pi f=12 \pi \mathrm{GHz}$ in both cases. The action-angle variables [25] are denoted $(J, \theta)$ and obtained through the canonical change of coordinates $x=2 J^{2} \sin ^{2} \varphi, p=J^{-1} \cot \varphi$ with $\theta=2 \varphi-\sin 2 \varphi$.

We assume $\omega=1$ without loss of generality (after a rescaling $H^{\prime}=\omega^{-2 / 3} H$, $t^{\prime}=\omega t, x^{\prime}=\omega^{2 / 3} x, p^{\prime}=\omega^{-1 / 3} p$ and consequently $\phi^{\prime}=\phi$ and $\left.F^{\prime}=\omega^{-4 / 3} F\right)$. The Hamiltonian (1) becomes [4]

$$
\begin{aligned}
H= & -\frac{1}{2 J^{2}}+2 J^{2}\left[F_{h} \sin (h t)\right. \\
& \left.+F_{l} \sin (l t+\phi)\right]\left(a_{0} / 2+\sum_{k=1}^{\infty} a_{k} \cos k \theta\right),
\end{aligned}
$$

where $a_{n}=\left[J_{n}(n)-J_{n-1}(n)\right] / n$ and $J_{n}$ 's are Bessel functions of the first kind. Note that for a given mode locking $h: l$, there are three variables $(J, \theta, t)$ and three parameters $\left(F_{h}, F_{l}, \phi\right)$. We denote this Hamiltonian $H\left(J, \theta, t ; F_{h}, F_{l}, \phi\right)$. In general, for a given set of parameter values, the phase space as depicted on a Poincaré section (a stroboscopic plot with period $2 \pi$, see Fig. 11), is composed of a mixture of regular structures surrounded by a chaotic sea. More precisely, the lower part of phase space $(J$ small $)$ is composed of rotational invariant tori (which persist from the integrable case $F_{h}=F_{l}=0$ as asserted by KAM theorem). The upper part is formed by regular islands surrounded by an unbounded chaotic sea. The ionizing trajectories are the ones in the chaotic sea which are unbounded ( $J$, or equivalently $n$, becomes progressively large). At the center of the regular islands, there are elliptic (stable) periodic orbits which organize the regular motion around them. These periodic orbits partly result from the break-up of resonant tori into a pair of elliptic/hyperbolic orbits (according to Birkhoff's theorem). Other periodic orbits result from the bifurcation of these orbits.

The general idea which is applied here is to follow a finite set of periodic orbits (both elliptic or hyperbolic) which have been identified as important. The criteria of choice combine several factors: the size of the island, the period and the location. For each periodic orbit of this set, we compute its location $(J, \theta)$ and its linear stability property as given by the residue (which is to be defined below). As the three parameters are varied, we follow the locations and residues of these orbits instead of computing the Poincaré section for each value of parameters. This allows us to have a clear idea of what is going on in phase space and to predict ionization thresholds. 
In what follows, we perform two kinds of computations : First, we compute the residue curves which are obtained as functions of the relative phase $\phi$ for fixed values of the amplitudes $\left(F_{h}, F_{l}\right)$. Second, we compute bifurcation surfaces which are defined as the set of parameters $\left(F_{h}, F_{l}, \phi\right)$ where a change of linear stability has occurred.

In order to start monitoring the stability of a family of periodic orbits, we first consider a specific periodic orbit, denoted $\mathcal{O}(0)$, of Hamiltonian (2) for $\phi=0$ which is our reference case. Numerically it is determined using a modified Newton-Raphson multi-shooting algorithm as described in Ref. [26]. The initial condition for launching the iterative algorithm can be taken from a Poincaré section, for instance. As $\phi$ is continuously varied, the orbit $\mathcal{O}(0)$ deforms continuously into $\mathcal{O}(\phi)$, whose period is denoted $T(\phi)$. In addition to its location, we also monitor its linear stability properties given by the integration of the reduced tangent flow

$$
\frac{d \mathcal{J}_{\phi}^{t}}{d t}=\mathbb{J} \nabla^{2} H(J, \theta, t ; \phi) \mathcal{J}_{\phi}^{t},
$$

where $\mathbb{J}=\left(\begin{array}{cc}0 & 1 \\ -1 & 0\end{array}\right)$ and $\nabla^{2} H$ is the two-dimensional Hessian matrix (composed of second derivatives of $H$ with respect to its canonical variables $J$ and $\theta$ ). The initial condition is $\mathcal{J}_{\phi}^{0}=\mathbb{I}_{2}$ (the two-dimensional identity matrix). The two eigenvalues of the monodromy matrix $\mathcal{J}_{\phi}^{T(\phi)}$ which make a pair $(\lambda(\phi), 1 / \lambda(\phi))$ determine the stability properties (the flow is volume preserving, thus the determinant of $\mathcal{J}_{\phi}^{T(\phi)}$ is equal to 1 ). The periodic orbit is elliptic if the spectrum is $\left(\mathrm{e}^{i \omega(\phi)}, \mathrm{e}^{-i \omega(\phi)}\right)$ (stable, except for some particular cases), or hyperbolic if the spectrum is $(\lambda(\phi), 1 / \lambda(\phi))$ with $\lambda(\phi) \in \mathbb{R}^{*}$ (unstable). The stability properties can be deduced in a concise form using Greene's residue $R$ [27, 28]

$$
R(\phi)=\frac{2-\operatorname{tr} \mathcal{J}_{\phi}^{T(\phi)}}{4}
$$

If $R(\phi) \in] 0,1[$, the periodic orbit is elliptic; if $R(\phi)<0$ or $R(\phi)>1$ it is hyperbolic; and if $R(\phi)=0$ and $R(\phi)=1$, it is parabolic. Generically, periodic orbits and their linear stabilities are robust against small changes of parameters, except at specific values where bifurcations occur [29]. These rare events affect the dynamical behavior drastically. They can be associated with an enhancement as well as a reduction of stability depending on the type of bifurcations, as shown in Refs. [24, 29]. We identify the bifurcations (if any) of a set of short periodic orbits, i.e., the type and the value of the parameter $\phi_{c}$ where they bifurcate. This provides a way to foretell if a relatively high ionization rate should be expected or not. The importance of considering two associated Birkhoff periodic orbits (i.e. periodic orbits with the same action but different angles in the integrable case, one elliptic and one hyperbolic), was emphasized in Ref. [24. The main reason is that it allows one to discard some specific bifurcations (like collisions or exchanges of stability) and draw appropriate conclusions concerning the enhancement or reduction of stability. 


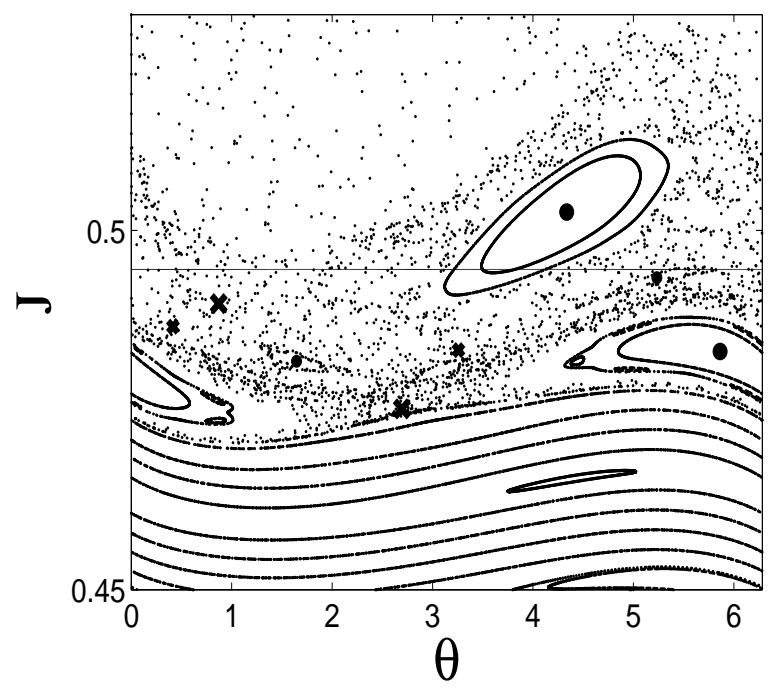

Figure 1. Poincaré section of Hamiltonian (2) for Case $(I)$ at $\phi=0$. Full big circles (or big crosses, respectively ) indicate the two elliptic (resp. hyperbolic) periodic orbits with period $2 \pi$ we consider. Full small circles ( or small crosses, respectively) indicate the two elliptic (resp. hyperbolic) periodic orbits with period $4 \pi$ we consider. The horizontal line corresponds to the principal quantum number $n=51$.

In the following sections, we analyze the short-time dynamics through residues of selected periodic orbits. We compute residue curves $\phi \mapsto R\left(\phi, F_{h}, F_{l}\right)$ for fixed values of the amplitudes $F_{h}$ and $F_{l}$, and bifurcation surfaces associated with a given periodic orbit defined as the set of parameters such that $R\left(\phi, F_{h}, F_{l}\right)=1$. We correlate these results with ionization probabilities obtained experimentally and also by quantum simulations through an empirical formula.

\section{3. $3: 1$ mode locking}

For this mode locking, we consider $F_{h}=24 \mathrm{Vcm}^{-1}$ and $F_{l}=53.4 \mathrm{Vcm}^{-1}$ as in Ref. [19]. These values correspond to the dimensionless values $F_{h}=24 v_{0}\left(t_{0} \omega\right)^{-4 / 3} / d_{0}=0.5278$ and $F_{l}=53.4 v_{0}\left(t_{0} \omega\right)^{-4 / 3} / d_{0}=1.1743$, where $v_{0}=0.036749326$ and $d_{0}=1.88972613 \times 10^{8}$ are both conversion factors, $t_{0}=2.4188843243 \times 10^{-17} \mathrm{~s}$ (atomic time unit). These values are referred as Case $(I)$ in what follows.

\subsection{Poincaré section}

Figure 1 shows a Poincaré section of Hamiltonian (2) for Case $(I)$ at $\phi=0$. The phase space is divided into two main parts : a lower regular containing many invariant tori, and a upper chaotic sea where trajectories escape rapidly to unbounded actions $J$ (ionized trajectories). We notice two main islands in the chaotic sea. At the centers of these islands sit elliptic periodic orbits with period $2 \pi$ (indicated by full circles). In addition, there is also a period 2 island in between these two main islands (associated 


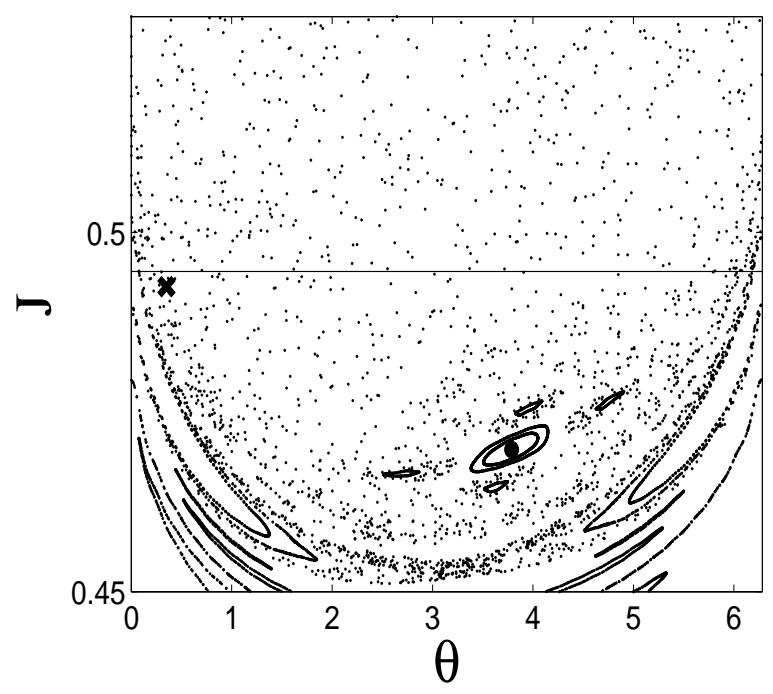

Figure 2. Poincaré section of Hamiltonian (2) for Case $(I)$ at $\phi=\pi / 3$. Full circle (respectively cross) indicates the elliptic (resp. hyperbolic) periodic orbit with period $2 \pi$ we consider. The horizontal line corresponds to the principal quantum number $n=51$.

with an elliptic and hyperbolic periodic orbits with period $4 \pi$ ). In the region of phase space around $J_{i} \approx 0.49$ (indicated by a straight line) where the initial states $n=51$ are prepared [19], any regular structures like the main islands and smaller ones are associated with trappings and hence reduce the ionization rate.

Figure 2 shows a Poincaré section of Hamiltonian (2) for Case $(I)$ at $\phi=\pi / 3$. Apparently the upper elliptic periodic orbit of period $2 \pi$ in Fig. 1 has disappeared in chaotic sea. This case is associated with a higher ionization probability. Therefore, the loss of stability of the main periodic orbit near $J_{i}$ plays a major role in the ionization process.

The residue method will monitor the location and stability of elliptic periodic orbits as well as their associated hyperbolic periodic orbits [24] as a function of the parameters of the system.

For Case $(I)$, Figure 3 shows the positions of the upper elliptic and hyperbolic periodic orbits of period $2 \pi$ on the Poincaré section as functions of $\phi$. We notice that the action $J$ is changing weakly as $\phi$ is varied. In contrast, its angle $\theta$ is very sensitive to this parameter.

\subsection{Residue curve}

We follow the residue for each of the elliptic and hyperbolic periodic orbits mentioned above as the parameter $\phi$ varies for Case $(I)$. Figure 4 shows the residue curves of these orbits.

In Fig. 4, we monitor the upper elliptic periodic orbit (period $2 \pi$ ) of Fig. 1 from $\phi=0$. This periodic orbit remains elliptic $(R(\phi) \in] 0,1[)$ until $\phi_{c} \approx 0.49$ where a 

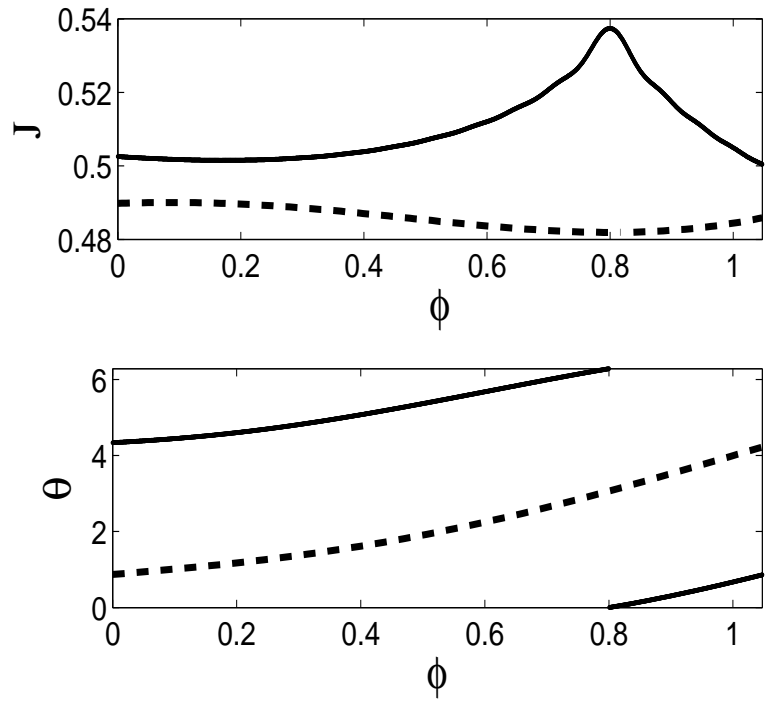

Figure 3. The positions of the upper elliptic and hyperbolic periodic orbits with period $2 \pi$ on the Poincaré section as functions of $\phi$ for Case $(I)$. The solid curves and the dashed ones correspond to the upper elliptic and hyperbolic periodic orbits of Fig. 11 respectively.

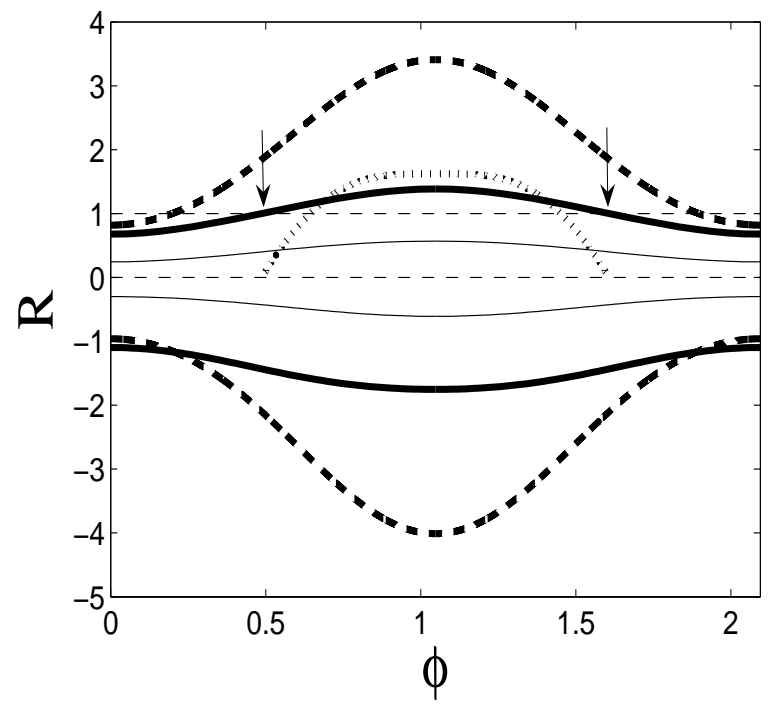

Figure 4. Residue curves for the four periodic orbits with period $2 \pi$ (solid curves) and the two periodic orbits with period $4 \pi$ (dashed bold curves), indicated by crosses and circles on Fig. 1 for Case $(I)$. The solid bold curves are for the upper set of elliptic/hyperbolic orbits of period $2 \pi$. Small arrows indicate where bifurcations happen. The dotted bold curve between two arrows is associated with the residues of the elliptic periodic orbit with period $4 \pi$ born of the period doubling bifurcation. 


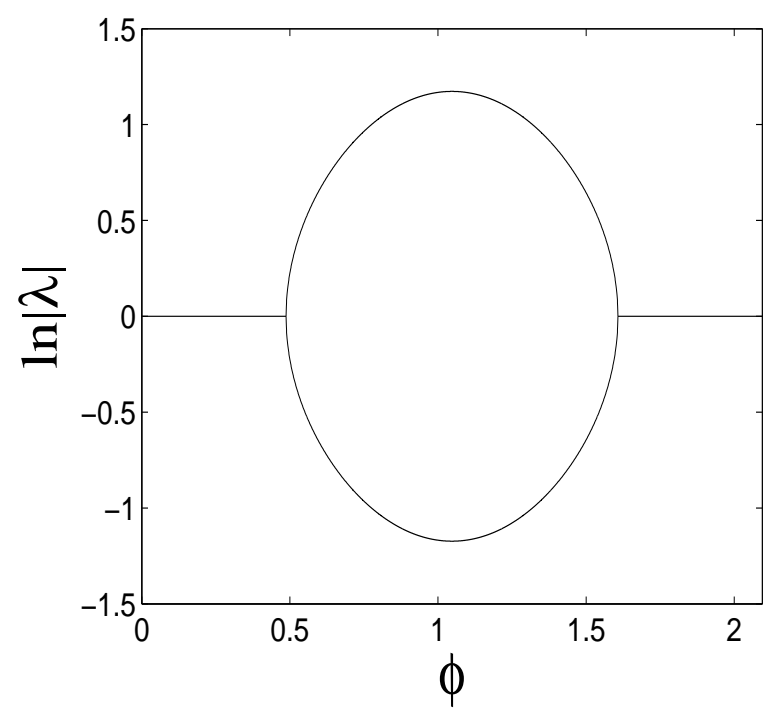

Figure 5. Bifurcation diagram for Case $(I)$ showing the bifurcations indicated by arrows in Fig. 4

bifurcation occurs. At this critical point, the orbit turns parabolic. Increasing $\phi$ further, it turns and remains hyperbolic $(R(\phi)>1)$ until $2 \pi / 3-\phi_{c}$ where another bifurcation appears, making the orbit elliptic again. This bifurcation process is of the period doubling kind at $\phi_{c}$ and a period halving at $2 \pi / 3-\phi_{c}$ (by symmetry).

The bifurcation diagram for Case $(I)$ is shown in Fig. 5. The computation of $\log \left|\lambda_{ \pm}(\phi)\right|$ (where $\lambda_{ \pm}(\phi)$ are the two eigenvalues of the monodromy matrix $\mathcal{J}_{\phi}^{T(\phi)}$ associated with the upper elliptic periodic orbit) gives $\log \left|\lambda_{ \pm}(\phi)\right|=0$ before the bifurcation and $\log \left|\lambda_{ \pm}(\phi)\right| \propto \pm \sqrt{\phi-\phi_{c}}$ right after the bifurcation.

Figure 6] shows a projection of the upper elliptic periodic orbit undergoing the period doubling bifurcation in $x-p$ representation where we note the doubling of the number of branches.

Note that while the upper elliptic periodic orbit undergoes a bifurcation as $\phi$ is varied, the other three periodic orbits with period $2 \pi$ retain the stability properties they had at $\phi=0$.

In the parameter range $\phi \in] \phi_{c}, 2 \pi / 3-\phi_{c}[$, the upper part of phase space does exhibit more chaos as it can be shown on the Poincaré section for $\phi=\pi / 3$ (see Fig. 2).

Since the initial atomic beam is taken in the region with principal quantum number $n=51$ which corresponds to action $J_{i} \approx 0.495$, the ionization rate is expected to be higher in the regime where there are no big islands in the chaotic sea, i.e. for $\phi \in] \phi_{c}, 2 \pi / 3-\phi_{c}[$. On a finer scale, one has to take into account the smaller regular structures that are present in the chaotic sea, like for instance the period-2 island on the Poincaré section (period $4 \pi$ ). A similar period doubling and halving occur at $\phi_{c, 1} \approx 0.199$ and $2 \pi / 3-\phi_{c, 1} \approx 1.895$ respectively, as can be seen on Fig. 四 (upper bold dashed curve). We notice that the residue curve for the period $4 \pi$ orbit is higher than the upper solid curve (for the period $2 \pi$ orbit) and the phase region between period 


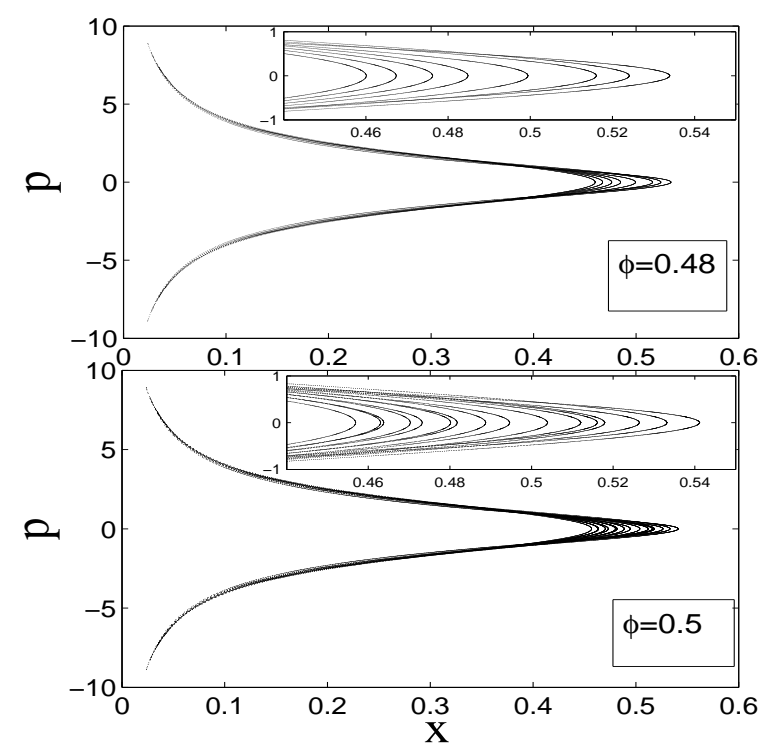

Figure 6. Upper elliptic periodic orbit (period $2 \pi$ ) of Case $(I)$ undergoing the periodic doubling bifurcation in $x-p$ representation. The insets shows the doubled number of branches indicating periodic doubling bifurcation.

doubling $\left(\phi_{c, 1}\right)$ and period halving $\left(2 \pi / 3-\phi_{c, 1}\right)$ is much wider than the one based on the period $2 \pi$. This observation indicates that for longer pulse duration for which longer periodic orbits have to be taken into account, the period $4 \pi$ orbit obtained by a repetition of the period $2 \pi$ orbit is more important (as a limiting factor) for ionization than the orbit with primary period $4 \pi$. As a by-product, the bifurcation at $\phi_{c, 1}$ does not affect significantly the leading ionization behavior based on period $2 \pi$. Another important periodic orbit with period $4 \pi$ is the one born of the period doubling bifurcation of the upper periodic orbit at $\phi_{c}$. This orbit is close to the newly hyperbolic periodic orbit and is at first elliptic, as seen on Fig. 4. It is expected that the associated elliptic islands slow down ionization. This orbit experiences a period doubling bifurcation at $\phi_{c, 2} \approx 0.643$ and halving bifurcation at $2 \pi / 3-\phi_{c, 2} \approx 1.451$. In summary, the shortest periodic orbits (period $2 \pi$ ) always play the most dominant role on ionizations rates, although with larger pulse durations longer periodic orbits might be taken into account for a more detailed analysis.

For values of $\phi$ around $\pi / 3$, a plateau is expected in the ionization probability versus $\phi$. The reason is that a strongly hyperbolic orbit only influences the ionization time and not the value of the ionization probability. Of course, this is true provided that the duration of the maximum pulse envelope is large enough. In the experiment, this is approximately 15 times the period of the shortest periodic orbits considered. Roughly speaking, this means that in the chaotic region all the orbits ionize (i.e., escape to a value of the action $J_{\text {ion }} \gtrsim 1.26$ ) regardless of the hyperbolicity degree. In Ref. [19], experimental results as well as one-dimensional quantum calculations show this plateau. From quantum calculations, $\phi_{c} \approx 0.5$ was obtained in Ref. [19] which is in very good agreement with the parameter value $\phi_{c} \approx 0.49$ at which the bifurcation of the upper 


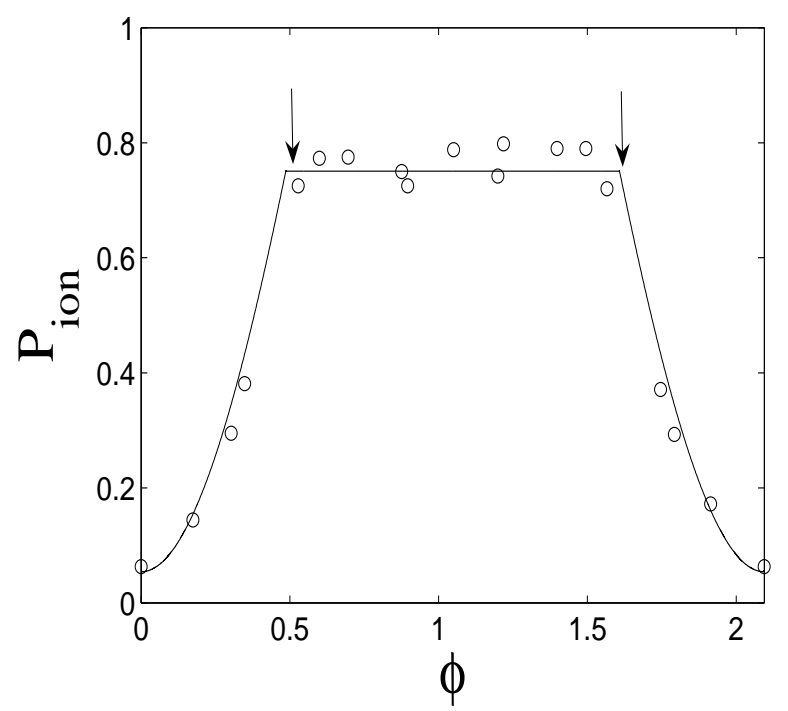

Figure 7. Normalized ionization probability vs $\phi$ based on Eq. (3) for Case (I) with $A=-2.52$ and $B=0.65$. Circles represent the data obtained by one-dimensional quantum calculations, taken from Ref. [19]. Only periodic orbits with period $2 \pi$ are considered.

elliptic periodic orbit (period $2 \pi$ ) occurs. If the duration of the experiment or simulations is longer (two or three times longer), then higher-order regular structures (like the regular island of period $4 \pi$ in the Poincaré section and the elliptic periodic orbit with period $4 \pi$ born of bifurcations) will play a role and we expect a similar, but smaller, plateau for $\phi \in] \phi_{c, 2}, 2 \pi / 3-\phi_{c, 2}[$.

The periodic orbit analysis above elucidates whether or not there is a significant ionization probability for specific parameter values, and also where plateaus are expected to occur. This qualitative agreement highlights the important role played by these orbits. Furthermore, we can obtain quantitative agreement concerning the shape of the ionization curve versus phase lag $\phi$ by using the residue curves. Here we devise an empirical formula for relative ionization probability in the following way : First, the values of $\phi$ giving the highest ionization would be the ones associated with the highest variations of the residues (in absolute value) with respect to the minimum ionization. Second, if the periodic orbit is too far (in action) from the considered action $J_{i}$ then it will not influence the dynamics so there should be a penalizing term depending on its position with respect to the chosen rescaled action. The relative ionization probability formula reads :

$$
P_{\text {ion }}(\phi)=A+B \sum_{m=1}^{M} \frac{\exp \left|R_{m}(\phi)-R_{m}\left(\phi_{0}\right)\right|}{\exp \left|\overline{J_{m}(\phi)}-J_{i}\right|},
$$

where the sum is taken over the $M$ different periodic orbits considered and $\overline{J_{m}(\phi)}=$ $\int_{0}^{2 \pi} J(\theta) d \theta / 2 \pi$ is the action of the periodic orbit $m$. The parameters $A$ and $B$ in Eq. (3) are merely a translation and a dilatation of the curve in order to match the mean value and the amplitude of variations of $P_{\text {ion }}$ obtained in Ref. [19]. This formula takes into 


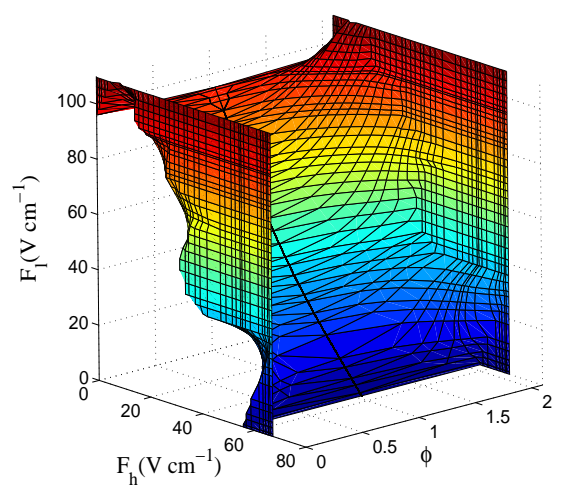

(a)

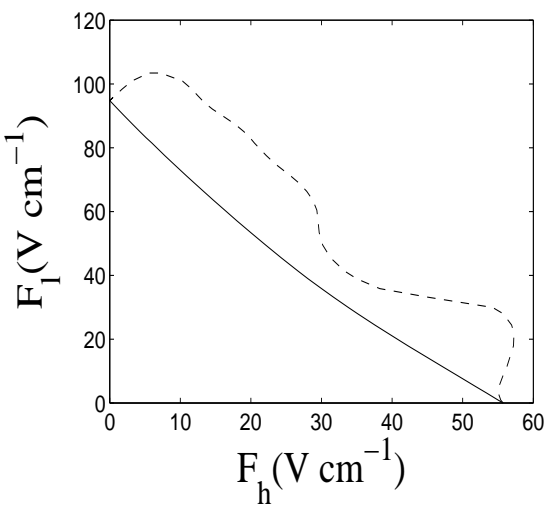

Figure 8. (color online). (a) Bifurcation surface in parameter space $\left(\phi, F_{h}, F_{l}\right)$ for $h: l=3: 1$. (b) The continuous curve is a section of the bifurcation surface (a) at $\phi=\pi / 3$, whereas the dashed one is for $\phi=1.95$.

account the value of the residues at $\phi_{0}$, where the minimum ionization takes place for each case. The aim is to set up a baseline for each of the periodic orbits (which is taken here at the value of the parameter where the ionization is minimal). Specifically for Case $(I) \phi_{0}=0$ [19]. In general, Eq. (3) can exhibit values which are greater than 1, which are not relevant. In order to remedy to this problem, we truncate $P_{\text {ion }}$ at the value where a bifurcation occurs in accordance with the previous discussion on the relevance of the degree of hyperbolicity. Therefore in the range where $R_{n}(\phi)$ is larger than one, $P_{\text {ion }}$ is constant (taken as the value of the residue at $\phi_{c}$ where the bifurcation occurs).

Figure 7 depicts $P_{\text {ion }}$ given by Eq. (3) versus parameter $\phi$ as well as the data taken from Ref. [19] for Case (I). Only the shortest periodic orbits with period $2 \pi$ are considered as relevant. We notice that the empirical formula reproduces accurately the results obtained from quantum calculations. If we take into account the period $4 \pi$ orbits for longer pulse duration, then a second plateau appears for $\phi \in] \phi_{c, 2}, 2 \pi / 3-\phi_{c, 2}[$ together with an increase at $\phi_{c, 1}$ and a decrease at $2 \pi / 3-\phi_{c, 1}$.

\subsection{Bifurcation surface}

The residue method is carried out to also predict the behavior of the system as all three parameters (the two amplitudes $F_{h}$ and $F_{l}$ and the phase lag $\phi$ ) are varied. In Fig. 8, we represent the set of parameters where the upper elliptic periodic orbit (with period $2 \pi$ ) of Fig. 1 is in fact parabolic (i.e., the set of parameters where the system undergoes a major bifurcation). The equation of this surface in parameter space is $R\left(\phi, F_{h}, F_{l}\right)=1$. The boundaries of the plateaus in parameter $\phi$ of Fig. 7 obtained by fixing the two values for $F_{h}$ and $F_{l}$ are on this surface. When $F_{h}$ approaches zero, this surface is less dependent on parameter $\phi$. Table 1 reports some values based on our analysis which are in good agreement with experimental results from Ref. [17]. Of course, the surface of Fig. 8 could also have been obtained from tedious numerical simulations of a large number of classical trajectories for each value of the parameters $\left(\phi, F_{h}, F_{l}\right)$. This 
Table 1. Ionization thresholds obtained for $F_{h}=6 \mathrm{Vcm}^{-1}$, experimentally in Ref. [17] and by the residue method (see Fig. 4). The 1f case corresponds to $F_{h}=0$.

\begin{tabular}{c|ccc}
\hline$F_{l}\left(\mathrm{Vcm}^{-1}\right)$ & $\phi=0$ & $\phi=\pi / 3$ & $1 \mathrm{f}$ \\
\hline 17 & 107 & 85 & 96 \\
residue & 109.6 & 81.4 & 94.8 \\
\hline
\end{tabular}

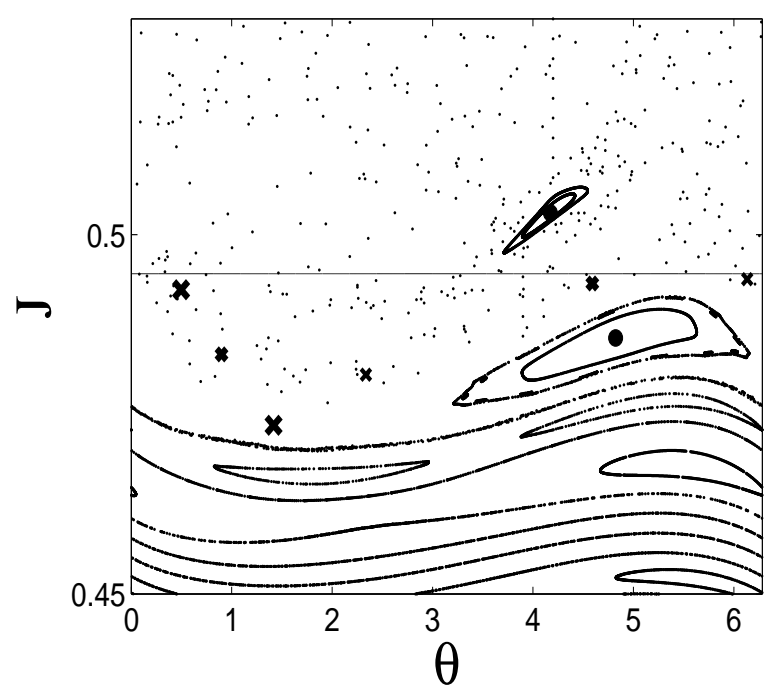

Figure 9. Poincaré section of Hamiltonian (2) for Case $(I I)$ at $\phi=0$. Full circles (respectively crosses) indicate the two elliptic (resp. hyperbolic) periodic orbits with period $2 \pi$ we consider. Small crosses indicate the two hyperbolic periodic orbits with period $4 \pi$ we consider. The horizontal line corresponds to the principal quantum number $n=51$.

integration needs to be performed for a sufficiently long time in order to decide if a given trajectory leads to ionization or not. In contrast, only one orbit for a short time (typically the period of the field) is needed for the residue analysis. Furthermore, using residues, this surface can be constructed locally without any need to consider all possible values of the parameters.

\section{3:2 mode locking}

In what follows, we study in detail one set of values for the amplitudes of the fields $F_{h}=25 \mathrm{Vcm}^{-1}$ and $F_{l}=33.5 \mathrm{Vcm}^{-1}$ which correspond to $F_{h}=0.5498$ and $F_{l}=0.7367$ in dimensionless units. This case will be referred as Case $(I I)$.

\subsection{Poincaré section}

Figure 9 shows a Poincaré section of Hamiltonian (2) for Case $(I I)$ at $\phi=0$. Similar to Case $(I)$, there are two primary islands in the chaotic sea where two elliptic periodic 


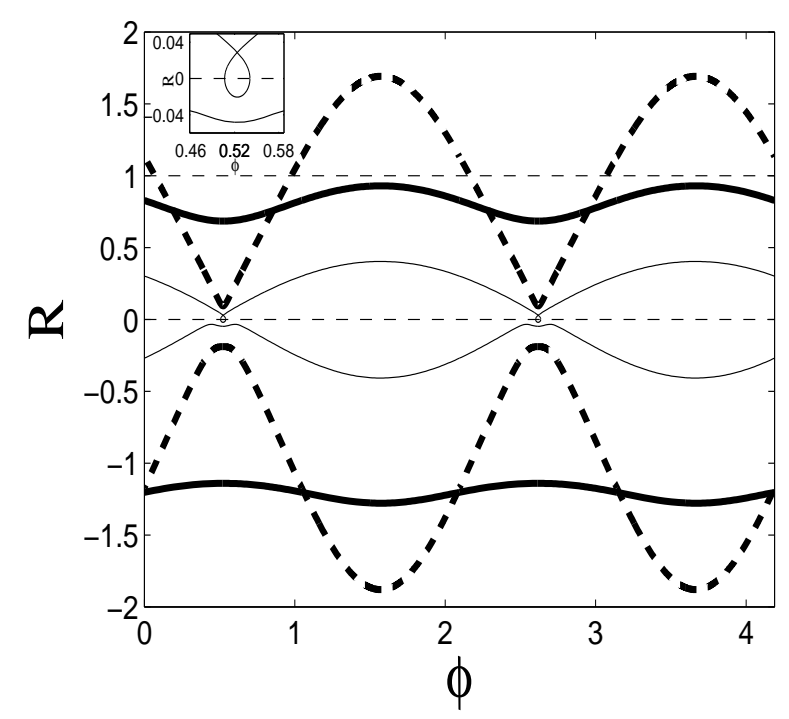

Figure 10. Residue curves for the four periodic orbits with period $2 \pi$ indicated by crosses and circles on Fig. 9 and the two periodic orbits indicated by small crosses with period $4 \pi$ for Case $(I I)$. The bold solid curves are for the upper set of elliptic/hyperbolic orbits with period $2 \pi$. The thin solid curves are for the lower set of elliptic/hyperbolic orbits with period $2 \pi$. The dashed curves are for the two initially hyperbolic periodic orbits with period $4 \pi$. The inset shows irregular behavior of residue at $\phi \simeq \pi / 6$. The same behavior also occurs at $\phi \simeq 5 \pi / 6$ for that residue curve.

orbits with period $2 \pi$ sit at the centers, and the two associated hyperbolic orbits are in the chaotic sea. In addition, there are also two associated hyperbolic periodic orbits with period $4 \pi$ as indicated by small crosses. One should notice that the (rescaled) principal quantum number considered in Ref. [19] lies in between these two islands (see the continuous horizontal line). We monitor the stability of this set of periodic orbits as we have performed for Case $(I)$.

\subsection{Residue curve}

Figure 10 shows the four residue curves of period $2 \pi$ orbits and the two residue curves of period $4 \pi$ orbits. For short periodic orbits (period $2 \pi$ ), the elliptic periodic orbits remain elliptic and the hyperbolic ones remain hyperbolic for all values of $\phi$. No bifurcation occurs except in a small range of phase (see inset of Fig. 10) where no significant stability change is observed. Consequently, the ionization probability is expected to be approximately independent of $\phi$ and to be lower than Case $(I)$ since for these values of amplitudes, the chaotic region is smaller. This is consistent with the experimental and quantum calculations of Ref. [19]. The experimental results show a nearly flat curve for the ionization probability versus $\phi$, whereas the quantum calculations show significant variations for this probability but no sharp increase and decrease as in Case $(I)$.

When the duration of pulse is longer we should take into account the effect of longer 


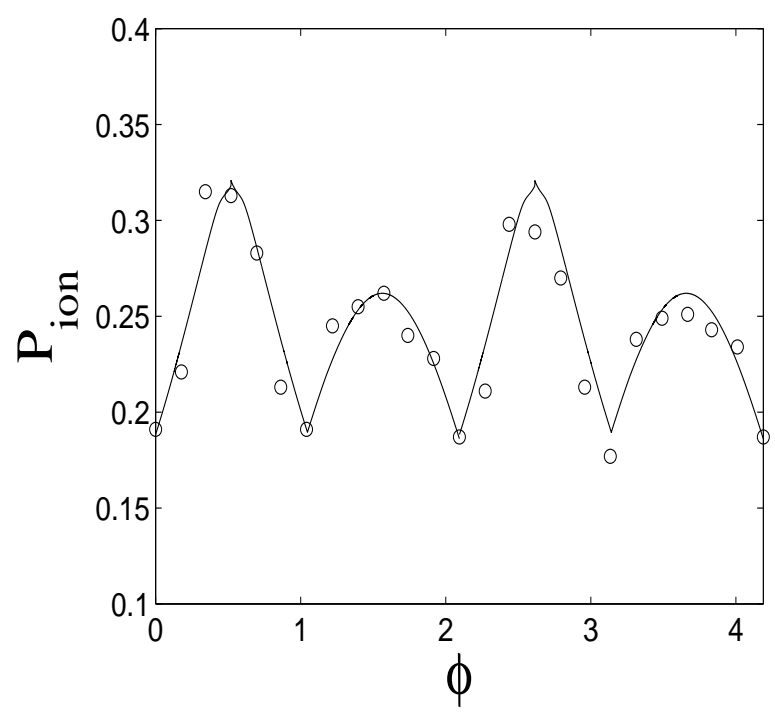

Figure 11. Normalized ionization probability vs $\phi$ based on Eq. (3) for Case (II) with $A=-0.485$ and $B=0.17$. Circles represent the data obtained by one-dimensional quantum calculations, taken from Ref. [19]. Only periodic orbits with period $2 \pi$ are considered.

periodic orbits whose residues are shown by bold dashed curves in Fig. 10 with period $4 \pi$ in this particular case. Apparently the upper period $4 \pi$ orbit experiences period halving bifurcations at $\phi \approx 0.07$ and $\phi \approx 2.164$; and period doubling bifurcations at $\phi \approx 0.977$ and $\phi \approx 3.072$.

Figure 11 depicts $P_{\text {ion }}$ given by Eq. (3) versus parameter $\phi$ as well as the data taken from Ref. 19] for Case $(I I)$. Only periodic orbits with period $2 \pi$ are considered. In Eq. (3) we again take $\phi_{0}=0$ since the ionization is minimal at $\phi=0$ for Case $(I I)$ [19]. We notice that it captures some essential features of the ionization curve, like the two unequal-sized peaks and the specific shape of both peaks (one more peaked, the other one, more round). This feature results from the asymmetry property of bichromatic microwave amplitude [30]. For this case, the bichromatic microwave field at $\phi=\pi / 6 \pm \triangle \phi$ has exactly the same amplitude but opposite direction as at $\phi=\pi / 2 \pm \triangle \phi$, where $0<\triangle \phi<\pi / 6$. Replacing "+" in front of the bichromatic field by "-" in Eq. (11) leads to a Hamiltonian for the other direction. Because of this symmetry property of bichromatic field, the ionization probability along the other direction can be obtained from Fig. 11 by a horizontal translation of $\pi / 3$. The total ionization rate should be the sum of the rates for both directions, which shows equally high peaks for total ionization rate in $\phi$. For longer pulse duration, we should consider longer periodic orbits like those with period $4 \pi$. Again we should take the baseline (minimal ionization point) at $\phi=0$. Since in Fig. 10 the residue value of elliptic periodic orbit with period $4 \pi$ (upper dashed curve) at minimal ionization point $(\phi=0)$ is already greater than 1 , the ionization rate should be high and roughly identical on the whole $\phi$ space. This indicates that when pulse duration gets longer, the ionization rate for any $\phi$ goes higher and suggests 
ionization rate for any $\phi$ will reach a roughly constant upper limit if the time duration is sufficiently long. This property is obviously true because when the system is exposed to external microwave field for a longer time, more electrons will gain sufficient energy to escape. However, as mentioned in Sec. 3, the shortest periodic orbits (period $2 \pi$ ) always play the most dominant role on ionizations rates, while the longer periodic orbits might be taken into account heuristically for a finer analysis when the pulse duration gets a lot longer. Finally, our analysis also indicates the asymmetric ionization property does not exist for 3:1 mode locking case, in agreement with Ref. [30].

\section{Generalization to $h: l$ mode locking}

In order to generalize this approach, we also investigate $h: l=2: 1$, denoted Case (III), and 5:1, denoted Case $(I V)$ mode locking.

\subsection{Residue curves}

For Case $(I I I)$, we take $F_{h}=24 \mathrm{Vcm}^{-1}, F_{l}=53.4 \mathrm{Vcm}^{-1}$ and the high frequency of $12 \mathrm{GHz}$. Figure 12 shows Poincaré section at $\phi=0$. It indicates the relevant periodic orbits to consider in the residue analysis. Figure 13 shows the residue curves based on the two periodic orbits with period $2 \pi$ in the chaotic sea of Fig. 12. No bifurcations take place for these orbits. Figure 14 depicts relative ionization rate with respect to $\phi$ based on Eq. (3)). For this case we take $\phi_{0}=0$ in Eq. (3) since the minimal ionization point is at $\phi=0$ according to the maximum field rule (see next section). Based on our approach, the asymmetric ionization property along a single direction (unequal-sized peaks) does appear for the $2: 1$ mode locking, in agreement with Ref. [30].

For Case $(I V)$, we take $F_{h}=24 \mathrm{Vcm}^{-1}, F_{l}=53.4 \mathrm{Vcm}^{-1}$ and the high frequency is $30 \mathrm{GHz}$. Figure 15 shows a Poincaré section at $\phi=0$, and Fig. [16 shows the residue curves based on the six periodic orbits of period $2 \pi$ in the chaotic sea of Fig. 15. We notice that the residue based on the upper elliptic periodic orbit has similar behavior to the one of the lower elliptic periodic orbit for Case $(I I)$ as shown in the inset of Fig. 10. The two residue curves (two thin lines) in Fig. 16 from the two lower periodic orbits in Fig. 15 are almost constant and therefore have little influence on ionization probability. Figure 17 depicts relative ionization rate with respect to $\phi$ based on Eq. (31) according to Fig. 16 (The lowest two periodic orbits are not considered since their corresponding residue curves are almost constant). For this case we take $\phi_{0}=\pi / 5$ in Eq. (3) since the minimal ionization point is at $\phi=\pi / 5$ according to the maximum field rule. The asymmetry ionization property does not exist for the 5:1 mode locking.

\subsection{Comparison with the maximum field rule}

A traditional way of explaining ionization probability behavior is the maximum field rule (peak field amplitude rule) formulated in Ref. [17, 31]. The relative ionization 


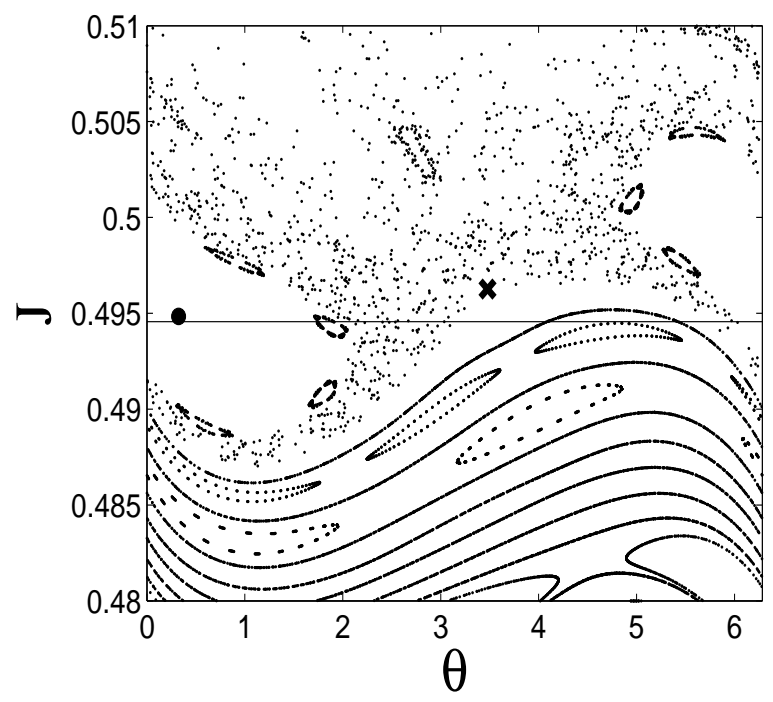

Figure 12. Poincaré section of Hamiltonian (2) for Case $(I I I)$ at $\phi=0$. Full circle (respectively cross) indicates the elliptic (resp. hyperbolic) periodic orbit with period $2 \pi$ we consider. The horizontal line corresponds to the principal quantum number $n=51$.

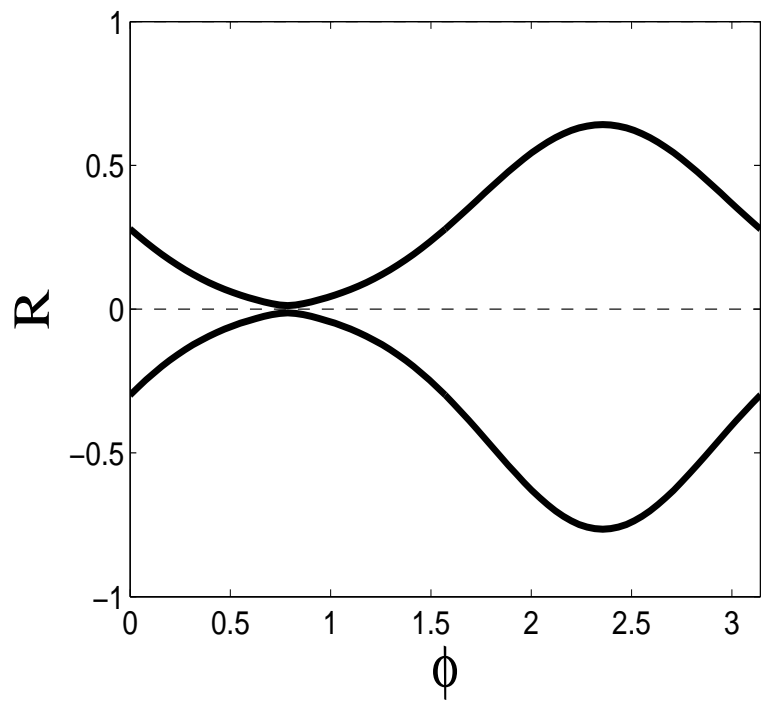

Figure 13. Residue curves for the two periodic orbits with period $2 \pi$ indicated by cross and circle on Fig. 12 for Case (III).

probability vs $\phi$ based on the maximum field rule can be written in the form

$$
P_{\text {ion }}(\phi)=C+D \max _{t \in[0,2 \pi]}\left|F_{h} \sin (h t)+F_{l} \sin (l t+\phi)\right| .
$$

The parameters $C$ and $D$ in Eq. (44) are merely a translation and a dilatation of the curve in order to make the curves from the maximum field rule comparable to those based on bifurcation analysis. Figure 18 shows relative ionization probability based on Eq. (4) for four different cases. Compared with our periodic orbit bifurcation analysis in 


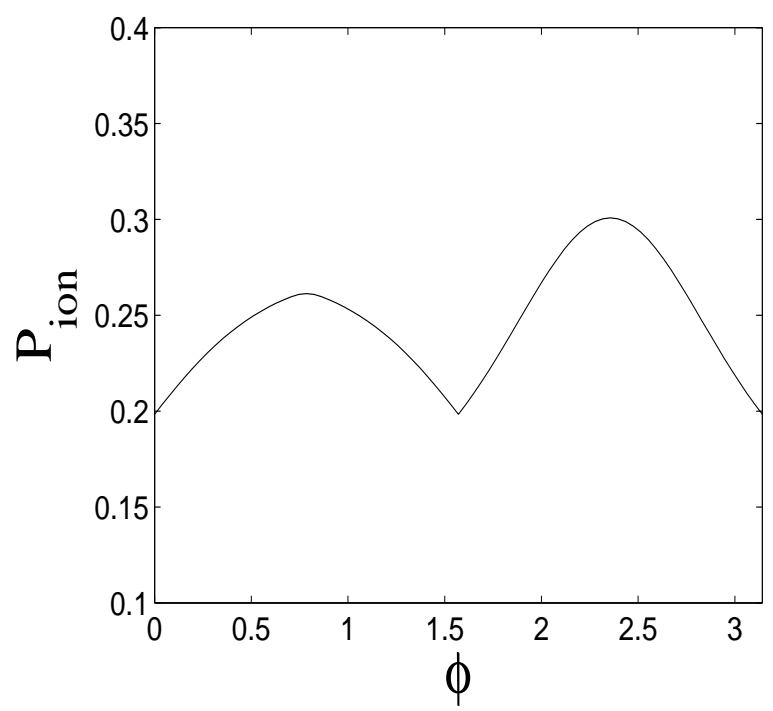

Figure 14. Relative ionization probability vs $\phi$ at principal quantum number $n=51$ based on Eq. (3) for Case (III) with $A=0$ and $B=0.1$. The absolute ionization rate may differ according to the pulse duration.

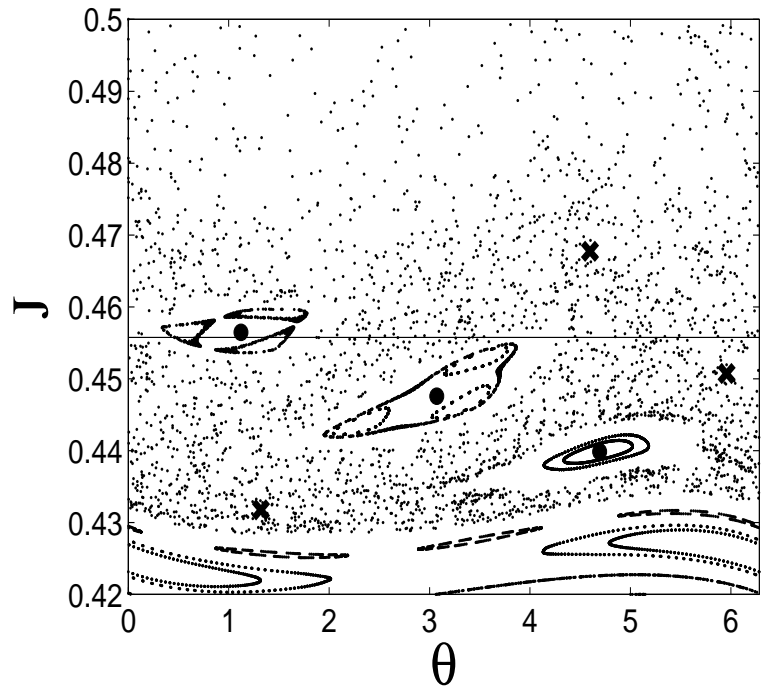

Figure 15. Poincaré section of Hamiltonian (2) for Case $(I V)$ at $\phi=0$. Full circles (respectively crosses) indicate the elliptic (resp. hyperbolic) periodic orbits with period $2 \pi$ we consider. The horizontal line corresponds to the principal quantum number $n=47$. 


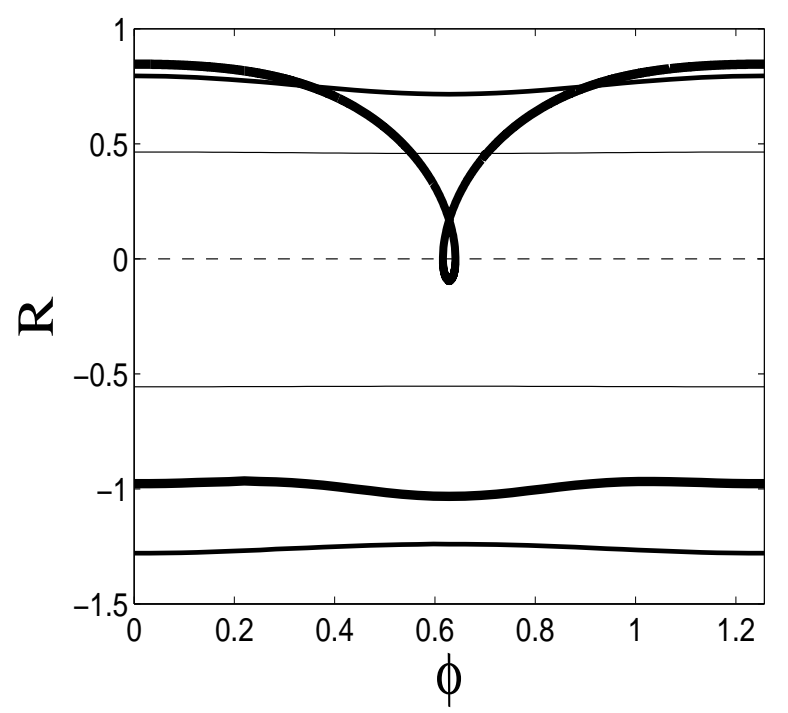

Figure 16. Residue curves of the six periodic orbits with period $2 \pi$ indicated by crosses and circles on Fig. 15] for Case $(I V)$.

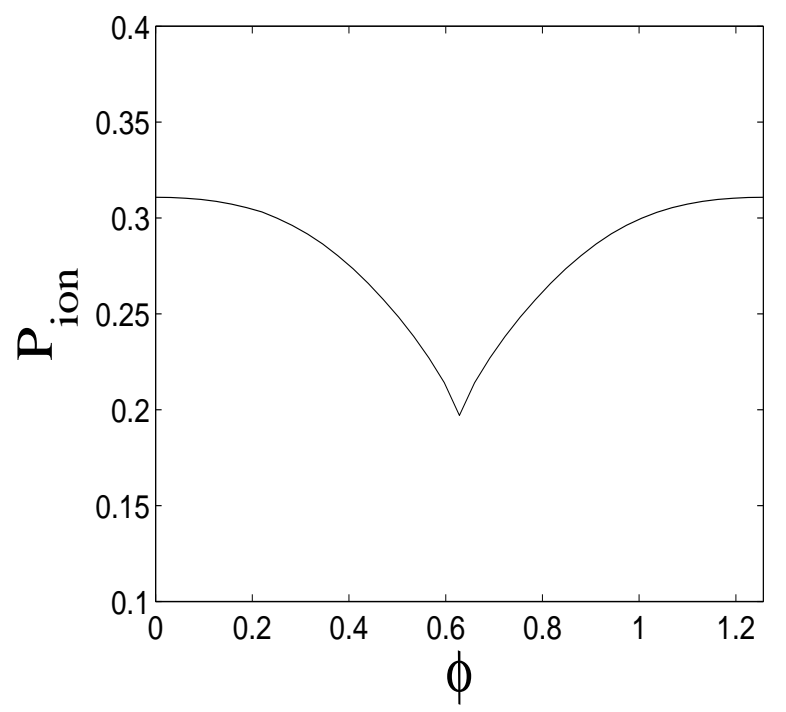

Figure 17. Relative ionization probability vs $\phi$ at principal quantum number $n=47$ based on Eq. (3) according to Fig. (16) for Case (IV) with $A=-0.2$ and $B=0.1$. The absolute ionization rate may differ according to the pulse duration.

finite pulse duration, apparently the maximum field rule does not result in quantitative agreement with the quantum simulation results of Ref. [19] for either Case $(I)$ or $(I I)$. In Case (III), the maximum field rule does not produce the two unequal-sized peaks as our bifurcation analysis does. Only in Case $(I V)$ do the results based on the maximum field rule agree with our bifurcation analysis because there is neither bifurcation nor asymmetric ionization property for this case. Insets of Fig. [18] show one cycle $(t \in[0,2 \pi])$ bichromatic field $\epsilon(t)=F_{h} \sin (h t)+F_{l} \sin (l t+\phi)$ with typical parameters as used for 
our previous analyses in dimensionless units for different $\phi$ values. Arrows indicate the $\phi$ values for which the bichromatic fields are drawn. Generally for Case $(I)$ the positive component of the bichromatic field is the same as the negative component for any $\phi$ in each full cycle (directional symmetry), whereas this directional symmetry does not appear for Case $(I I I)$ [30] except for some specific $\phi$ values like $\phi=0, \frac{\pi}{2}, \pi$. Similarly, for Case $(I I)$ this directional symmetry generally does not appear except for some specific $\phi$ values like $\phi=0, \frac{\pi}{3}, \frac{2 \pi}{3}, \pi, \frac{4 \pi}{3}$, whereas for Case $(I V)$, this directional symmetry does appear for any $\phi$ values. Although the maximum field rule can be used to determine qualitative features like the minimal ionization point with respect to $\phi$, the quantitative agreement is not as satisfactory as the one given by a method which relies on analyzing the chaotic dynamics like the one used in this article based on periodic orbit bifurcation analysis to depict relative ionization.

\section{Acknowledgements}

This research was supported by the US National Science Foundation. C.C. acknowledges support from Euratom-CEA (contract EUR 344-88-1 FUA F). We thank Xavier Leoncini and Luca Perotti for useful discussions.

\section{References}

[1] R. Blümel and W.P. Reinhardt, Chaos in Atomic Physics (Cambridge University Press, Cambridge, UK, 1997).

[2] J.E. Bayfield and P.M. Koch, Phys. Rev. Lett. 33, 258 (1974).

[3] B.I. Meerson, E.A. Oks and P.V. Sasorov, Pis'ma Zh. Eksp. Teor. Fiz. 29, 79 (1979) [JETP Lett. 29, 72 (1979)].

[4] G. Casati, B.V. Chirikov, I. Guarneri and D.L. Shepelyansky, Phys. Rep. 154, 77 (1987).

[5] R.V. Jensen, S.M. Susskind and M.M. Sanders, Phys. Rep. 201, 1 (1991).

[6] P.M. Koch and K.A.H. van Leeuwen, Phys. Rep. 255, 289 (1995).

[7] H. Rabitz, R. de Vivie-Riedle, M. Motzkus and K. Kompa, Science 288, 824 (2000).

[8] M. Shapiro and P. Brumer, Phys. Rep. 425, 195 (2006).

[9] L. Ko, M.W. Noel and T.F. Gallagher, J. Phys. B 32, 3469 (1999).

[10] F. Ehlotzky, Phys. Rep. 345, 175 (2001).

[11] J.E. Howard, Phys. Lett. A 156, 286 (1991).

[12] A. Haffmans, R. Blümel, P.M. Koch and L. Sirko, Phys. Rev. Lett. 73, 248 (1994).

[13] A. Buchleitner, D. Delande and J.-C. Gay, J. Opt. Soc. Am. B 12, 505 (1995).

[14] D. Petrosyan and P. Lambropoulos, Phys. Rev. Lett. 85, 1843 (2000).

[15] M. Ivanov, P.B. Corkum, T. Zuo and A.D. Bandrauk, Phys. Rev. Lett. 74, 2933 (1995).

[16] L. Sirko, S.A. Zelazny, and P.M. Koch, Phys. Rev. Lett. 87, 043002 (2001).

[17] L. Sirko and P.M. Koch, Phys. Rev. Lett. 89, 274101 (2002).

[18] V. Batista and P. Brumer, Phys. Rev. Lett. 89, 143201 (2002).

[19] P.M. Koch, S.A. Zelazny and L. Sirko, J. Phys. B 36, 4755 (2003).

[20] C. Rangan, A.M. Bloch, C. Monroe and P.H. Bucksbaum, Phys. Rev. Lett. 92, 113004 (2004).

[21] V. Constantoudis and C.A. Nicolaides, J. Chem. Phys. 122, 084118 (2005).

[22] G. G. Carlo, G. Benenti, G. Casati and D. L. Shepelyansky, Phys. Rev. Lett. 94, 164101 (2005).

[23] S. Huang, C. Chandre and T. Uzer, J. Phys. B 40, F181 (2007)

[24] R. Bachelard, C. Chandre and X. Leoncini, Chaos 16, 023104 (2006). 


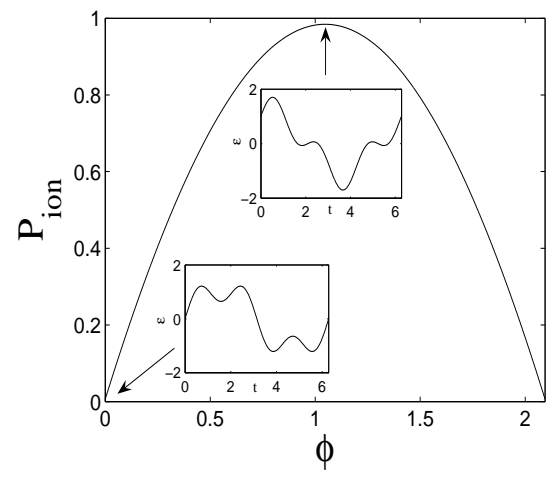

(a)

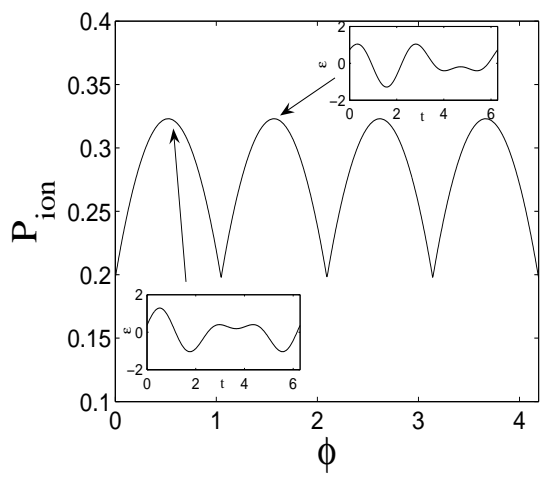

(b)

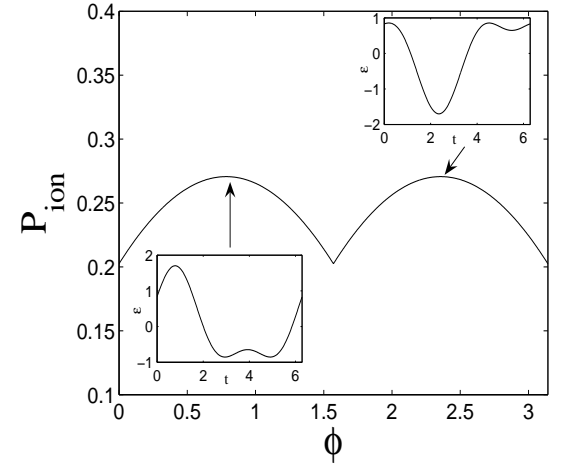

(c)

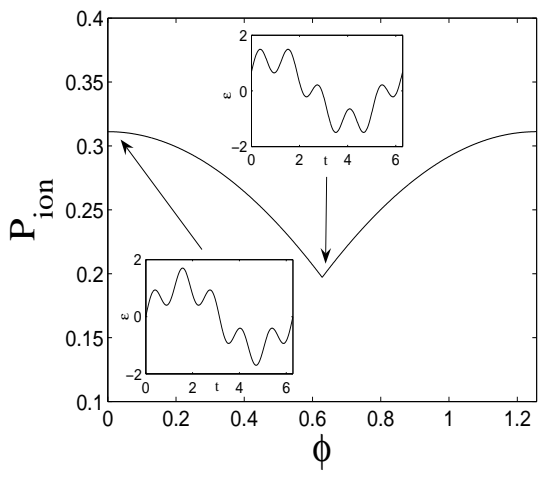

(d)

Figure 18. Relative ionization probability based on the maximum field rule, on Eq. (4), for $(a)$ Case $(I)$ with $C=-2.42$ and $D=2$. Insets show the bichromatic field for $\phi=0$ (bottom panel) and $\phi=\pi / 3$ (top panel).(b) Case (II) with $C=-2.25$ and $D=2$. Insets show the bichromatic field for $\phi=\pi / 6$ (bottom panel) and $\phi=\pi / 2$ (top panel). (c) Case (III) with $C=-0.24$ and $D=0.3$. Insets show the bichromatic field for $\phi=\pi / 4$ (bottom panel) and $\phi=3 \pi / 4$ (top panel). and (d) Case $(I V)$ with $C=-0.625$ and $D=0.55$. Insets show the bichromatic field for $\phi=0$ (bottom panel) and $\phi=\pi / 5$ (top panel). Arrows indicate the $\phi$ values for which the bichromatic fields 
[25] J.G. Leopold and D. Richards, J. Phys. B 18, 3369 (1985).

[26] P. Cvitanović, R. Artuso, R. Mainieri, G. Tanner and G. Vattay, Chaos: Classical and Quantum, ChaosBook. org (Niels Bohr Institute, Copenhagen 2005).

[27] J.M. Greene, J. Math. Phys. 20, 1173 (1979).

[28] R.S. MacKay, Nonlinearity 5, 161 (1992).

[29] J.R. Cary and J.D. Hanson, Phys. Fluids 29, 2464 (1986).

[30] K.J. Schafer and K.C. Kulander, Phys. Rev. A 45, 8026 (1992).

[31] B.E. Sauer, S. Yoakum, L. Moorman, P.M. Koch, D. Richards, and P.A. Dando, Phys. Rev. Lett. 68, 468 (1992). 